\title{
Exploration of horizontal intrinsic spin resonances with two partial Siberian snakes
}

\author{
F. Lin, ${ }^{1,2}$ L. A. Ahrens, ${ }^{1}$ M. Bai, ${ }^{1}$ K. Brown, ${ }^{1}$ E. D. Courant,${ }^{1}$ C. Gardner, ${ }^{1}$ J. W. Glenn, ${ }^{1}$ H. Huang,,${ }^{1}$ S. Y. Lee, ${ }^{2}$ \\ A. U. Luccio, ${ }^{1}$ W. W. MacKay, ${ }^{1}$ V. Ptitsyn, ${ }^{1}$ T. Roser, ${ }^{1}$ J. Takano, ${ }^{1,3}$ S. Tepikian, ${ }^{1}$ N. Tsoupas, ${ }^{1}$ A. Zelenski, ${ }^{1}$ and K. Zeno ${ }^{1}$ \\ ${ }^{1}$ Brookhaven National Laboratory, Upton, New York 11973, USA \\ ${ }^{2}$ Indiana University, Bloomington, Indiana 47405, USA \\ ${ }^{3}$ RIKEN, Wako, Saitama 351-0198, Japan \\ (Received 22 February 2007; published 13 April 2007)
}

\begin{abstract}
Two partial Siberian snakes were used to avoid all the spin imperfection and vertical intrinsic resonances in the alternating gradient synchrotron (AGS) at Brookhaven National Laboratory. However, the horizontal betatron motion can cause polarization loss resulting from the nonvertical stable spin direction in the presence of two partial snakes. This type of resonance, called a horizontal intrinsic spin resonance, was observed and systematically studied in the AGS. A simplified analytic model and numerical simulation have been developed to compare with experimental data. Properties of the horizontal intrinsic resonance are discussed.
\end{abstract}

DOI: 10.1103/PhysRevSTAB.10.044001

\section{INTRODUCTION}

The spin motion in a synchrotron is governed by the Thomas-BMT equation [1],

$$
\frac{d \vec{S}}{d t}=\frac{e}{\gamma m} \vec{S} \times\left[(1+G \gamma) \vec{B}_{\perp}+(1+G) \vec{B}_{\|}\right]
$$

where the spin vector $\vec{S}$ is in the particle rest frame and the magnetic fields are in the laboratory frame. The unperturbed spin precession tune [2] is $\nu_{s}=G \gamma$, where $G \approx$ 1.7928 is the proton anomalous gyromagnetic g-factor and $\gamma=\frac{E}{m c^{2}}$ is the Lorentz factor. The spin vector that is invariant under the one-turn map (OTM) [2] is called the stable spin direction or the spin closed orbit $\hat{n}_{\mathrm{co}}$. Any spin vector not lying on $\hat{n}_{\text {co }}$ will precess around $\hat{n}_{\text {co }}$ with $\nu_{s}$ spin precessions per revolution.

For a planar accelerator, the stable spin direction is normally in the vertical direction. The horizontal field can perturb the spin vector away from the vertical and cause spin polarization loss when the spin precession frequency equals the frequency of the spin perturbing magnetic fields. Two important types of spin resonances are the imperfection resonance driven by the vertical closed orbit errors in quadrupoles, and the vertical intrinsic resonance driven by the vertical betatron motion in quadrupoles. The imperfection resonances happen at $G \gamma=n$, and the intrinsic resonances happen at $G \gamma=k P \pm \nu_{y}$ [2], where $n$ and $k$ are integers, $P$ is the superperiodicity of the lattice, and $\nu_{y}$ is the vertical betatron tune.

The final polarization $P_{f}$ after passing through an isolated spin resonance is given by the Froissart-Stora formula [3],

$$
\frac{P_{f}}{P_{i}}=2 e^{-\left[\left(\pi|\epsilon|^{2}\right) /(2 \alpha)\right]}-1,
$$

where $P_{i}$ is the polarization before crossing the resonance,
PACS numbers: 29.27.Bd, 29.27.Hj, 41.75.Ak, 07.77.Ka

$\epsilon$ is the resonance strength, and $\alpha=\frac{d(G \gamma)}{d \theta}$ is the resonance crossing rate. The intrinsic resonance strength is proportional to the square root of the particle emittance $I$,

$$
|\epsilon(I)|^{2}=\left|\epsilon\left(I_{0}\right)\right|^{2} \frac{I}{I_{0}},
$$

where $I_{0}$ is the rms emittance of the beam, and $\epsilon\left(I_{0}\right)$ is the rms value of resonance strength. For a beam with Gaussian distribution $\rho(I)=\frac{1}{2 I_{0}} e^{-\left[I /\left(2 I_{0}\right)\right]}$ in the phase space, the beam polarization after passing through an intrinsic resonance is given by

$$
\left\langle\frac{P_{f}}{P_{i}}\right\rangle=\int_{0}^{\infty}\left[2 e^{-\left[\left(\pi|\epsilon|^{2}\right) /(2 \alpha)\right]}-1\right] \rho(I) d I,
$$

or

$$
\left\langle\frac{P_{f}}{P_{i}}\right\rangle=\frac{1-\frac{\pi\left|\epsilon\left(I_{0}\right)\right|^{2}}{\alpha}}{1+\frac{\pi\left|\epsilon\left(I_{0}\right)\right|^{2}}{\alpha}} .
$$

The final polarization strongly depends on the rms resonance strength $\epsilon\left(I_{0}\right)$. For the alternating gradient synchrotron (AGS), the strong intrinsic resonances can cause partial spin flip [4].

In order to overcome the spin resonances, partial snakes [5-7] have been employed in the AGS during the polarized proton acceleration. Snakes are local spin rotators that cause the spin vector to precess by an angle of less than or equal to $180^{\circ}$ around an axis in the horizontal plane. Historically, a combination of a 5\% normal conducting solenoid partial snake plus an AC dipole were used to overcome imperfection and four strong intrinsic resonances [8] in the AGS. The polarization of $40 \%$ was achieved. In 2004, a 5.9\% normal conducting helical dipole partial snake (called warm snake) [9] has been installed to reduce transverse coupling resonances caused by the strong solenoidal field of the solenoid snake [10]. With 
the combined operation of the warm snake and the $\mathrm{AC}$ dipole, 55\% polarization was achieved at the AGS extraction energy. However, the goal of full polarization transmission requires the correction of all the intrinsic resonances including the weak ones. In addition to the $5.9 \%$ warm snake, a superconducting helical dipole snake (called cold snake) $[11,12]$ with a maximum strength of $25 \%$ at the AGS extraction energy was added in the AGS before the polarized proton run in 2005 to overcome all the imperfection and the vertical intrinsic resonances. With the two snakes separated by $1 / 3$ of the ring, the spin tune is given by [11]

$$
\begin{aligned}
\nu_{s}= & \frac{1}{\pi} \arccos \left(\cos \frac{\chi_{c}}{2} \cos \frac{\chi_{w}}{2} \cos [G \gamma \pi]\right. \\
& \left.-\sin \frac{\chi_{c}}{2} \sin \frac{\chi_{w}}{2} \cos \left[G \gamma \frac{\pi}{3}\right]\right)
\end{aligned}
$$

where $\chi_{c}, \chi_{w}$ are the spin rotation angles caused by the cold and warm snake, respectively.

The OTM of the spin transfer matrix becomes

$$
\begin{aligned}
T= & e^{-i(1 / 2) G \gamma[2 \pi-(2 \pi / 3)-\theta] \sigma_{3}} e^{-i\left(\chi_{w} / 2\right) \sigma_{2}} e^{-i(1 / 2) G \gamma(2 \pi / 3) \sigma_{3}} \\
& \times e^{-i\left(\chi_{c} / 2\right) \sigma_{2}} e^{-i(1 / 2) G \gamma \theta \sigma_{3}},
\end{aligned}
$$

where $\theta$ is the orbital angle between the measurement location and the cold partial snake. The OTM can also be expressed as

$$
T=e^{-i \pi \nu_{s} \hat{n}_{\mathrm{co}} \cdot \vec{\sigma}},
$$

where $\nu_{s}$ is the spin tune and $\hat{n}_{\text {co }}$ is the stable spin direction or the spin closed orbit given by

$$
\hat{n}_{\mathrm{co}}=\cos \alpha_{1}{\hat{e_{1}}}+\cos \alpha_{2}{\hat{e_{2}}}+\cos \alpha_{3} \hat{e_{3}},
$$

where $\left(\cos \alpha_{1}, \cos \alpha_{2}, \cos \alpha_{3}\right)$ are the spin closed orbit directional cosines along the radially outward, longitudinally forward, and vertically transverse axes, respectively. Comparing matrix elements of the OTM in Eqs. (7) and (8), we obtain

$$
\begin{aligned}
\cos \alpha_{1}= & \frac{-1}{\sin \pi \nu_{s}}\left\{\cos \frac{\chi_{w}}{2} \sin \frac{\chi_{c}}{2} \sin [G \gamma(\pi-\theta)]\right. \\
& \left.+\sin \frac{\chi_{w}}{2} \cos \frac{\chi_{c}}{2} \sin \left[G \gamma\left(\frac{\pi}{3}-\theta\right)\right]\right\}, \\
\cos \alpha_{2}= & \frac{1}{\sin \pi \nu_{s}}\left\{\cos \frac{\chi_{w}}{2} \sin \frac{\chi_{c}}{2} \cos [G \gamma(\pi-\theta)]\right. \\
& \left.+\sin \frac{\chi_{w}}{2} \cos \frac{\chi_{c}}{2} \cos \left[G \gamma\left(\frac{\pi}{3}-\theta\right)\right]\right\}, \\
\cos \alpha_{3}= & \frac{1}{\sin \pi \nu_{s}}\left(\cos \frac{\chi_{w}}{2} \cos \frac{\chi_{c}}{2} \sin [G \gamma \pi]\right. \\
& \left.-\sin \frac{\chi_{w}}{2} \sin \frac{\chi_{c}}{2} \sin \left[\frac{G \gamma}{3} \pi\right]\right) .
\end{aligned}
$$

Equation (6) shows that, with two partial snakes configuration, the deviation of spin tune from an integer reaches its maximum every $G \gamma=3 n$, where $n$ is an integer. Since the AGS has a superperiodicity of 12 and the vertical betatron tune is close to integer 9, this feature provides the maximum space for placing the vertical betatron tune in the prohibited region of spin tune at all the strong vertical intrinsic resonances [11]. The stop band at all the other integers is large enough for avoiding all the weak vertical intrinsic resonances. Equation (12) also shows that the tilt of the stable spin direction away from vertical direction reaches its minimum at $G \gamma=3 n+1.5$. In the AGS, the injection energy is at $G \gamma=4.5$, which makes the polarization loss due to the spin direction mismatch minimum [11].

Figure 1 gives the spin tune from Eq. (6) (top plot) and vertical component of the stable spin direction Eq. (12) (bottom plot) as a function of $G \gamma$ with two partial snakes separated by $1 / 3$ of the ring in the AGS. The two snakes run at constant fields, which results in the snake strength dropping when the beam energy ramps up. The partial snake percentage quoted in this paper is the strength at top energy. Two conditions of snake strengths are presented, one for $14 \%$ cold snake and 5.9\% warm snake, one for $10 \%$ cold snake and $5.9 \%$ warm snake. The imperfection and vertical intrinsic resonances have been overcome successfully in the AGS.

With the two partial snakes the stable spin direction is not vertical. Therefore the perturbing fields that rotate the spin away from the stable direction have vertical as well as horizontal components. Particles undergoing horizontal betatron oscillations encounter vertical field deviations at

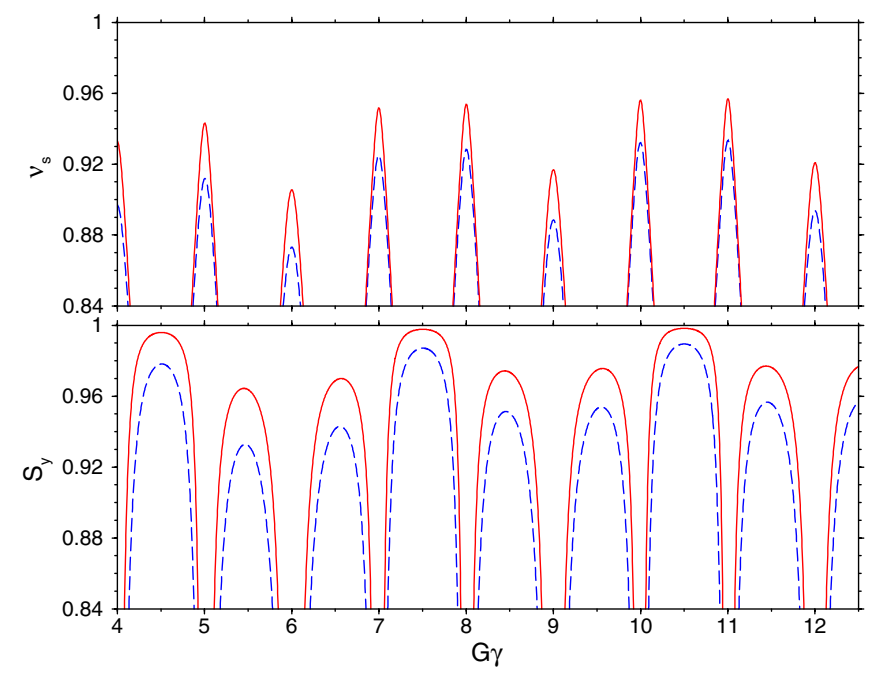

FIG. 1. (Color) The spin tune from Eq. (6) (top plot) and vertical component of the stable spin direction Eq. (12) (bottom plot) as a function of $G \gamma$ with two partial snakes separated by $1 / 3$ of the ring in the AGS. The solid curves are for $10 \%$ cold snake and $5.9 \%$ warm snake, the dashed ones are for $14 \%$ cold snake and $5.9 \%$ warm snake. 
the horizontal oscillation frequency. As a result, resonances with the spin tune are driven by the horizontal betatron oscillations, and will occur whenever the spin tune satisfies $\nu_{s}=k \pm \nu_{x}$. This type of resonance is called "horizontal intrinsic resonance." Since the two partial snakes are constructed by the helical dipole magnet field, the vertical magnet fields distributed along the snakes are the main parts of the polarization perturbation.

This paper studies the effect of horizontal resonances on the polarization. We organize our paper as follows. Section II gives simulation results, both from a simplified analytic model and from multiparticle spin tracking. Section III presents the experimental method and results on the exploration of the horizontal intrinsic resonance.

\section{ANALYTIC MODEL AND SIMULATION RESULTS}

Polarization loss due to the horizontal motion was studied both by simplified analytic mode and by the spin tracking program SPINK [13].

The simplified analytic model was based on Thomas Roser's estimate of the resonance strength of each of the resonances occurring whenever $\nu_{s}=n \pm \nu_{x}$; if, as is the case, these resonances are very narrow and well separated, the overall depolarization factor is just the product of the effective Froissart-Stora factors for each of the resonances as shown in Eq. (13):

$$
\frac{P_{f}}{P_{i}}=\prod_{n(\gamma)} \frac{1-\frac{\pi|\epsilon(\gamma)|^{2}}{\alpha}}{1+\frac{\pi|\epsilon(\gamma)|^{2}}{\alpha}},
$$

where the product is over all the values of $\gamma$ where the resonance occurs. In this simple model, the horizontal betatron motion is represented by a single kick at the strong cold snake. The full horizontal oscillations in the rest of the ring have no effect on the spin since the spin precession commutes with the precession in the main dipoles. Note that this model therefore predicts that horizontal resonances do not depend on the superperiodicity of the lattice but only depend on the lattice function at the snake. The horizontal resonance strength is given by the product of the horizontal component of the stable spin direction $P_{h}(G \gamma)$ calculated from the OTM and the rms value of the deviation of the total horizontal orbit angle $\Theta$ :

$$
\epsilon(\gamma)=\frac{G \gamma}{2 \pi} P_{h}(G \gamma) \Theta=K(\gamma) \sqrt{\gamma}
$$

where $\Theta=\sqrt{\frac{\left(1+\alpha_{x}^{2}\right) I_{0 x}}{\gamma \beta_{x}}} \sin \left(\pi \nu_{x}\right), I_{0 x}$ is the normalized rms horizontal emittance, $\nu_{x}$ is the horizontal betatron tune, and $\alpha_{x}$ and $\beta_{x}$ are the Courant-Snyder parameters in front of the cold snake. $K(\gamma)$ is a weakly energy dependent factor since the lattice functions have some energy dependence. The effect of a second weaker snake will undulate the resonance strength depending on the spin precession between the two snakes. However, on average over many resonances the effect will cancel and is therefore ignored in this model.

The spin tracking program SPINK was used with an ensemble of 100 particles with a Gaussian distribution in the horizontal phase space and a rms momentum spread $\frac{\Delta p}{p}$ of 0.001 . The realistic AGS lattice and acceleration conditions including the varied acceleration rate and the varied strengths of the two partial snakes along the energy ramp were used in the tracking. The final polarization was calculated as the average polarization of the 100 particles projected to the stable spin direction.

Figure 2 shows both simplified analytic model and spin tracking results for two partial snakes separated by $1 / 3$ of the ring with two different snake strength setups: the top plot is for the $10 \%$ cold snake and the $5.9 \%$ warm snake, the bottom one for the $14 \%$ cold snake and the $5.9 \%$ warm snake. Zero vertical emittance was used to eliminate the residual polarization effect from the vertical motion and the horizontal betatron tune was set at 8.72. In general, the polarization should stay constant or decrease gradually if the spin motion is completely incoherent between resonance crossing. However, the spin tracking shows the spin coherence, which means the spin components after one resonance are affected by the resonances they passed. As a result, the spin tracking gives less polarization loss than the simplified analytic model.

In order to compare to the simplified analytic model that treats the horizontal resonances as isolated resonances, one

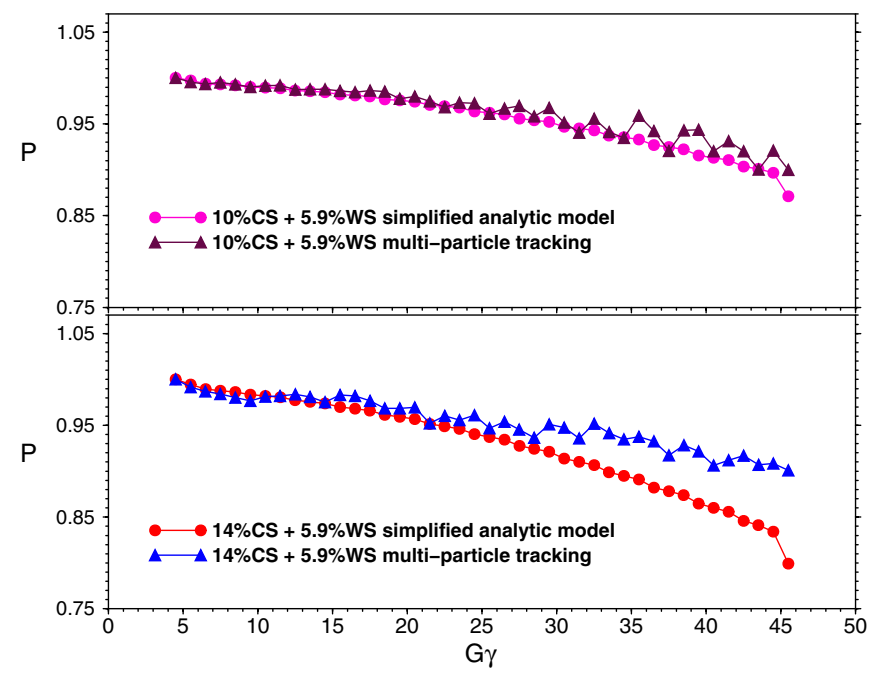

FIG. 2. (Color) Simplified analytic model and multiparticle tracking with a Gaussian distribution of particles in the horizontal phase space. The upper plot is for $10 \%$ and $5.9 \%$ partial snakes; the lower one is for $14 \%$ and $5.9 \%$ partial snakes. Both simplified analytic model and simulation have the same normalized rms horizontal emittance, zero vertical emittance, and 8.72 horizontal tune. The multiparticle tracking shows that the spin coherence results in less polarization loss compared to the simplified analytic model. 


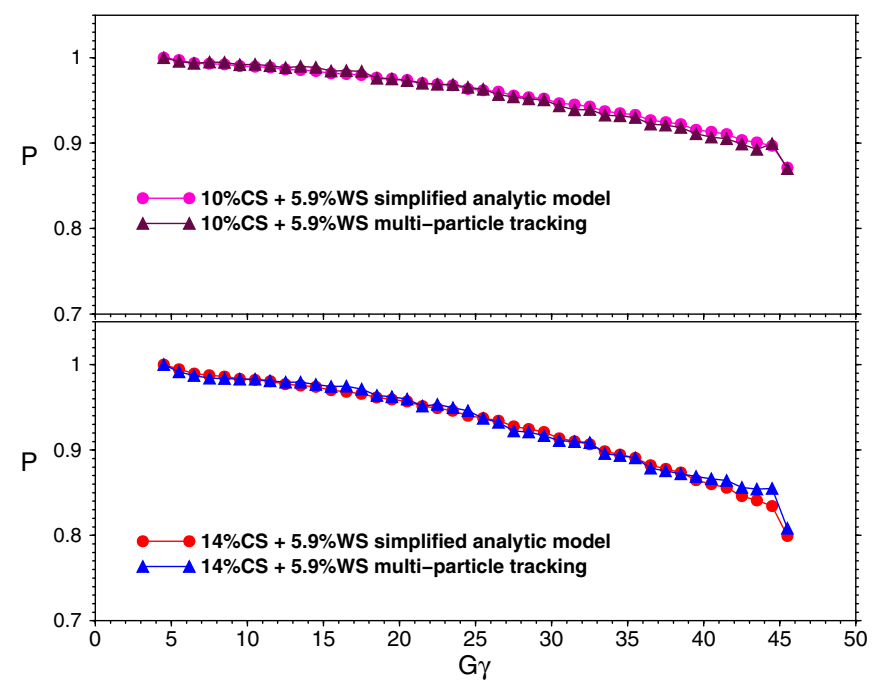

FIG. 3. (Color) Spin tracking from both simplified analytic model and multiparticle with Gaussian distribution. The upper plot is for $10 \%$ and $5.9 \%$ partial snakes; the lower one is for $14 \%$ and $5.9 \%$ partial snakes. Both simplified analytic model and simulation have the same normalized rms horizontal emittance, zero vertical emittance, and 8.72 horizontal tune. The spin coherence is artificially removed from the multiparticle tracking.

approach has been carried out to remove the spin coherence effect in the multiparticle tracking: the tracking has been taken in a short energy range and restarted tracking by putting the spin direction along the stable spin direction. With this change, the polarization from the two models agrees very well as shown in Fig. 3, the top plot is for the $10 \%$ cold snake and the $5.9 \%$ warm snake, the bottom one for the $14 \%$ cold snake and the $5.9 \%$ warm snake. This result implies that the horizontal resonances are indeed dominated by the spin kick at the cold partial snake and the discrepancy of the spin tracking from the simplified analytic model in Fig. 2 is due to the spin coherence. With this agreement, the $K(\gamma)$ value can be predicted from Eq. (14) given the snake strength and normalized emittance.

\section{EXPERIMENTAL RESULTS}

In 2006, experiments were carried out in the AGS to explore horizontal resonance. The AGS accelerates polarized protons from 2.4 to $24 \mathrm{GeV}$. Two partial snakes were installed to maintain the beam polarization. The cold snake is located at $30^{\circ}$ bending angle after the injection point, and the warm snake is located at $150^{\circ}$ bending angle after the injection point. The apparatus used for measuring polarization is the Coulomb nuclear interference (CNI) polarimeter [14] located at $82.5^{\circ}$ bending angle after the injection point. With the proton-carbon elastic scattering, the beam polarization at the top energy was determined by measuring the left-right asymmetry in the horizontal scattering plane.
As shown in Eqs. (3) and (5), higher polarization is expected for the particles with smaller emittance. Hence, a polarization profile in the horizontal plane, namely, different polarization for particles with different horizontal emittance, can develop due to the numerous horizontal spin resonances. This horizontal polarization profile was measured by placing the vertically oriented carbon target at different horizontal positions in the beam.

Figure 4 shows both the horizontal beam profiles and the horizontal polarization profile measurements for different cold snake strengths of $10 \%$ (the solid lines) and $14 \%$ (the dashed lines), respectively. The warm snake strength was set $5.9 \%$ for both cases. For a fair comparison between the two profile measurements, the beam intensity of $1.0 \times$ $10^{11}$ protons/bunch and the beam size were kept similar.

Beam profiles were fitted using the Gaussian distribution,

$$
\rho(x)=A e^{-\left[\left(x-x_{0}\right)^{2} /\left(2 \sigma^{2}\right)\right]},
$$

where $A$ is the amplitude, $x_{0}$ is the beam center position, and $\sigma$ is the rms beam size. They are determined by fitting the experimental data.

In order to derive the polarization based on the fitted rms beam size and the beam center position obtained from the beam profile, the following assumptions were assumed: (i) depolarization effects due to the vertical coupling motion do not show on the horizontal plane, (ii) beam only crossed 82 isolated horizontal intrinsic resonances during the acceleration based on Eq. (13), (iii) the horizontal resonance strength is proportional to the square root of the normalized horizontal beam emittance. Since we can only measure the polarization vs the horizontal position,

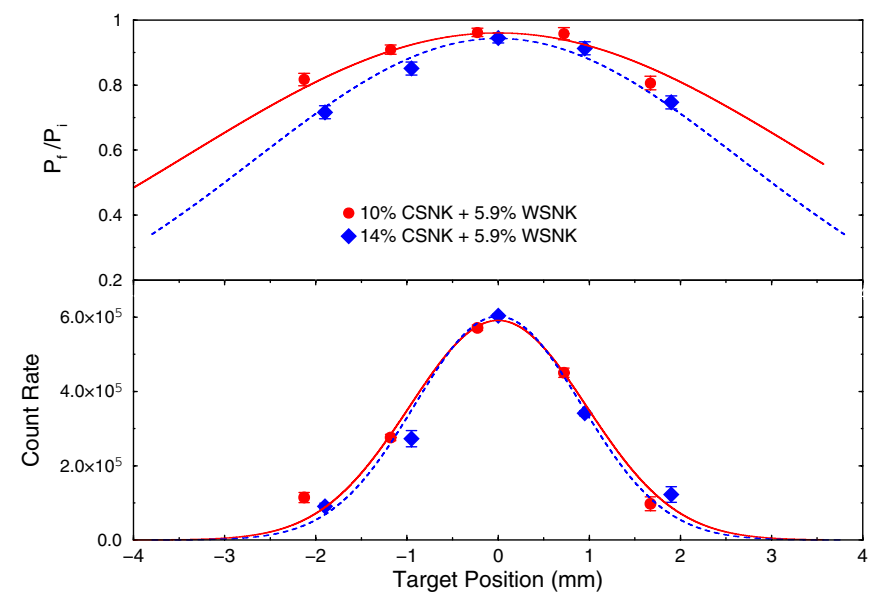

FIG. 4. (Color) The polarization profiles (upper) and horizontal beam profiles (bottom) in the AGS for two different strengths of the partial snakes. The solid curves are for $10 \%$ cold snake and $5.9 \%$ warm snake, and the dashed ones for $14 \%$ cold snake and $5.9 \%$ warm snake. The beam profile curves are fitted using Eq. (15) and polarization profile curves are fitted using Eq. (18) after crossing 82 isolated horizontal intrinsic resonances. 
we need to average the beam polarization over the conjugate phase-space coordinate:

$$
\begin{aligned}
\left\langle\frac{P_{f}}{P_{i}}\right\rangle= & \int_{0}^{\infty}\left[2 e^{-\left[\left(\pi K^{2} \gamma\right) /(2 \alpha)\right]\left[\left(x^{2}+p_{x}^{2}\right) / \sigma^{2}\right]}-1\right] \\
& \times \frac{1}{\sqrt{2 \pi} \sigma} e^{-\left[p_{x}^{2} /\left(2 \sigma^{2}\right)\right]} d p_{x},
\end{aligned}
$$

where $p_{x}$ is the phase-space coordinate conjugate to $x$, and Eq. (3) is replaced by

$$
|\epsilon(I)|^{2}=\left|\epsilon\left(I_{0}\right)\right|^{2} \frac{I}{I_{0}}=K^{2} \gamma \frac{\left(x^{2}+p_{x}^{2}\right)}{\sigma^{2}},
$$

where $\left(x, p_{x}\right)$ are the normalized conjugate phase-space coordinates, and $I=x^{2}+p_{x}^{2}$. The energy independent factor $K=\frac{\epsilon(\gamma)}{\sqrt{\gamma}}$ is introduced in order to make a comparison with the simplified analytic model. After the integral over $p_{x}$ and crossing the $n$ isolated resonances, we obtain

$$
\left\langle\frac{P_{f}}{P_{i}}\right\rangle=\prod_{n(\gamma)}\left[2 e^{-\left\{\left[\pi K^{2} \gamma\left(x-x_{0}\right)^{2}\right] /\left[\alpha(\gamma) 2 \sigma^{2}\right]\right\}} \sqrt{\frac{1}{\frac{\pi K^{2} \gamma}{\alpha(\gamma)}+1}}-1\right],
$$

where $P_{f}$ is the measured final polarization after the acceleration, and $P_{i}$ is the fitted initial polarization at injection. The relative polarizations shown in Fig. 4 are the values of $P_{f}$ divided by the $P_{i} . \alpha(\gamma)$ is the acceleration rate for the AGS. $x_{0}$ and $\sigma$ are determined by fitting. The energy independent factor $K$ from fitting the polarization profile is $(2.171 \pm 0.15) \times 10^{-5}$ for the $10 \%$ cold snake and the $5.9 \%$ warm snake, $(2.606 \pm 0.14) \times 10^{-5}$ for the $14 \%$ cold snake and the $5.9 \%$ warm snake. The error bar is from polarization statistical errors only. As expected, a stronger partial snake gives stronger horizontal spin resonance and leads to more curvature in the polarization profile as shown on the polarization profile in Fig. 4.

The averaged weak energy dependent factor $K(\gamma)$ can be calculated from the simplified analytic model. In order to get the rms beam size $\sigma_{\beta}$ due to the betatron oscillation, the measured beam size should subtract the part due to dispersion:

$$
\sigma_{\beta}^{2}=\sigma^{2}-\left(D_{x} \frac{\Delta p}{p}\right)^{2}
$$

where $\sigma$ is given from the measured rms beam profile, $D_{x}=1.66 \mathrm{~m}$ is the dispersion at the location of polarimeter, and $\frac{\Delta p}{p}=0.0003$ is the momentum spread of the beam at the measurement energy. The normalized rms horizontal emittance $\epsilon_{N \text { rms }}$ is obtained by

$$
\epsilon_{N \mathrm{rms}}=\beta \gamma \epsilon_{\mathrm{rms}}=\beta \gamma \frac{\sigma_{\beta}^{2}}{\beta_{x}} .
$$

Here $\beta, \gamma$ are the Lorentz factors, and $\beta_{x}$ is the horizontal betatron amplitude at CNI polarimeter. Therefore, the normalized rms horizontal emittance was $1.79 \mu \mathrm{m}$ for the $10 \%$ cold snake and $1.46 \mu \mathrm{m}$ for the $14 \%$ cold snake. Calculated from Eq. (14), the averaged $K(\gamma)$ is $2.571 \times$ $10^{-5}$ and $3.358 \times 10^{-5}$, respectively. The simplified model also shows that the stronger the snake strength, the stronger the horizontal resonance. Although the simplified model does not agree well with the real lattice tracking result quantitatively, it is useful in understanding effects of the horizontal resonances.

\section{CONCLUSION}

With two partial snakes in the AGS, experimental data revealed the existence of horizontal intrinsic resonances. Spin tracking also confirmed this observation and the resonance strength can be estimated from the experimental data. The horizontal polarization profile was measured to explore the horizontal resonance in the AGS. The experimental data fitting gives the rms horizontal resonance energy independent factor $K$ as $(2.171 \pm 0.15) \times 10^{-5}$ for the $10 \%$ cold snake and the $5.9 \%$ warm snake and as $(2.606 \pm 0.14) \times 10^{-5}$ for the $14 \%$ cold snake and the $5.9 \%$ warm snake. Both of them are close to the predicted averaged $K(\gamma)$ values from the simplified analytic model: $2.571 \times 10^{-5}$ and $3.358 \times 10^{-5}$, respectively. The resonance strength strongly depends on the total strength of the partial snakes. Without artificially removing the spin coherence, the spin tracking shows less polarization loss, which has better agreement with the experimental result. To overcome horizontal intrinsic resonances, the horizontal betatron tune has to be put into the spin tune gap generated by the two partial snakes. This requires an upgrade of AGS quadrupole power supplies.

\section{ACKNOWLEDGMENTS}

The authors would like to thank John Laster, Joe Skelly, and Kin Yip for their help in the tunemeter, IPM, and polarimeter software work. The authors would also like to thank all coordinators and operators in the main control room for their help in the machine tuning, all engineers and technicians who have contributed to the polarized proton development. The work was funded by NSF PHY-0552389 and DOE DE-FG02-92ER40747 and DE-AC02-98CH1886, and with support of RIKEN (Japan) and Renaissance Technologies Corp. (U.S.A.).

[1] L. H. Thomas, Philos. Mag. 3, 1 (1927); AIP Proc. 95, 4 (1982); V. Bargmann, L. Michel, and V. L. Telegdi, Phys. Rev. Lett. 2, 435 (1959).

[2] S. Y. Lee, Spin Dynamics and Snakes in Synchrotrons (World Scientific, Singapore, 1997). 
[3] M. Froissart and R. Stora, Nucl. Instrum. Methods 7, 297 (1960).

[4] F.Z. Khiari et al., Phys. Rev. D 39, 45 (1989).

[5] Ya. S. Derbenev and A. M. Kondratenko, Sov. Phys. Dokl. 20, 562 (1976).; Ya. S. Derbenev et al., Part. Accel. 8, 115 (1978).

[6] T. Roser, AIP Conf. Proc. 187, 1442 (1989).

[7] H. Huang et al., Phys. Rev. Lett. 73, 2982 (1994); H. Huang, Ph.D. thesis, Indiana University, 1995.

[8] M. Bai et al., Phys. Rev. Lett. 80, 4673 (1998).

[9] J. Takano et al., IEEE Trans. Appl. Supercond. 14, 457 (2004).
[10] C. Ohmori et al., Phys. Rev. Lett. 75, 1931 (1995)

[11] T. Roser et al., Proceedings of EPAC 2004, Lucerne, Switzerland, 2004, p. 1577; Proceedings of SPIN 2004, Trieste, Italy, 2004, p. 687.

[12] E. Willen et al., Proceedings of PAC 2005, Knoxville, Tennessee, 2005, p. 2935.

[13] A. U. Luccio, Proceedings of the Adriatico Research Conference on Trends in Collider Spin Physics, Trieste, Italy, 1995, p. 235.

[14] J. Tojo et al., Phys. Rev. Lett. 89, 052302 (2002); J. J. Wood, Ph.D. dissertation, UCLA, 2005. 